\title{
TWO-STREAM-LIKE INSTABILITY IN DILUTE HOT RELATIVISTIC BEAMS AND ASTROPHYSICAL RELATIVISTIC SHOCKS
}

\author{
Ehud NaKar ${ }^{1}$, Antoine BreT ${ }^{2,3}$, And Miloš Milosavljević ${ }^{4}$ \\ ${ }^{1}$ Raymond and Beverly Sackler School of Physics \& Astronomy, Tel Aviv University, Tel Aviv 69978, Israel \\ ${ }^{2}$ ETSI Industriales, Universidad de Castilla-La Mancha, 13071 Ciudad Real, Spain \\ ${ }^{3}$ Instituto de Investigaciones Energéticas, 13071 Ciudad Real, Spain \\ ${ }^{4}$ Department of Astronomy and Texas Cosmology Center, University of Texas, 1 University Station C1400, Austin, TX 78712, USA \\ Received 2011 March 14; accepted 2011 June 17; published 2011 August 16
}

\begin{abstract}
Relativistic collisionless shocks are believed to be efficient particle accelerators. Nonlinear outcome of the interaction of accelerated particles that run ahead of the shock, the so-called precursor, with the unperturbed plasma of the shock upstream, is thought to facilitate additional acceleration of these particles and to possibly modify the hydrodynamic structure of the shock. We explore here the linear growth of kinetic modes appearing in the precursor-upstream interaction in relativistic shocks propagating in non- and weakly magnetized plasmas: electrostatic two-stream parallel mode and electrostatic oblique modes. The physics of the parallel and oblique modes is similar, and thus, we refer to the entire spectrum of electrostatic modes as "two-stream-like." These modes are of particular interest because they are the fastest growing modes known in this type of system. Using a simplified distribution function for a dilute ultrarelativistic beam that is relativistically hot in its own rest frame, yet has momenta that are narrowly collimated in the frame of the cold upstream plasma into which it propagates, we identify the fastest growing mode in the full $k$-space and calculate its growth rate. We consider all types of plasma (pairs and ions-electrons) and beam (charged and charge-neutral). We find that unstable electrostatic modes are present in any type of plasma and for any shock parameters. We further find that two modes, one parallel $\left(k_{\perp}=0\right)$ and the other one oblique $\left(k_{\perp} \sim k_{\|}\right)$, are competing for dominance and that either one may dominate the growth rate in different regions of the phase space. The dominant mode is determined mostly by the perpendicular spread of the accelerated particle momenta in the upstream frame, which reflects the shock Lorentz factor. The parallel mode becomes more dominant in shocks with lower Lorentz factors (i.e., with larger momentum spreads). We briefly discuss possible implications of our results for external shocks in gamma-ray burst sources.
\end{abstract}

Key words: acceleration of particles - cosmic rays - plasmas - shock waves

Online-only material: color figures

\section{INTRODUCTION}

Cosmic rays are believed to be accelerated in astrophysical collisionless shocks. The leading acceleration process is diffusive shock acceleration (DSA; e.g., Bell 1978; Blandford \& Ostriker 1978; Blandford \& Eichler 1987), where charged particles are accelerated by crossing the shock back and forth. Thus, an integral element of this picture is a precursor of accelerated particles that runs ahead of the shock and interacts with the incoming, and mostly unperturbed, upstream plasma. The upstream plasma must interact with the precursor in such a way as to deflect the accelerated particles back into the shock and thus close the Fermi cycle and enable farther acceleration. It also provides an early perturbation to the upstream plasma that may, through a transfer of energy and momentum, and through an excitation of electromagnetic perturbations in the plasma, modify the shock structure (e.g., Blandford 1980; Drury \& Voelk 1981). If a different acceleration mechanism is at work (e.g., shock-drift acceleration; Webb et al. 1983), then the accelerated particles should similarly cross the shock into the upstream, thus producing a precursor, but these particles are not necessarily deflected back into the shock.

The general picture described above should apply to both relativistic and non-relativistic shocks and in various types of upstream plasma and precursors, independently of the plasma magnetization level and composition. The details of the precursor interaction, however, will depend on the specific system properties. Here, we explore a family of such systems that are thought to occur in astrophysical environments: relativistic collisionless shocks that propagate into unmagnetized or weakly magnetized plasma of various compositions. Until recently, theoretical understanding of particle acceleration in such shocks was based mostly on analytic or semianalytic prescriptions (e.g., Achterberg et al. 2001; Ellison \& Double 2002; Niemiec \& Ostrowski 2006, and references therein), in which the structure of the medium that scatters the accelerated particles was assumed ad hoc. A major breakthrough was achieved recently, when two-dimensional first-principles particle-in-cell (PIC) simulations of unmagnetized pair and ion-electron shocks provided direct evidence for the acceleration of particles via DSA and for the formation of a shock precursor (Spitkovsky 2008a, 2008b; Keshet et al. 2009; Martins et al. 2009). As expected, the numerical simulations show that a precursor of accelerated particles runs ahead of the shock and interacts, by exciting collective modes, with the initially undisturbed upstream plasma. While opening a new window into the physical processes that take place in these shocks, PIC simulations are still rather limited in size and duration and an analytic treatment of the processes that shape the shock is still required to extrapolate between the simulations and real astrophysical systems. Moreover, existing simulations have not converged to a steady state shock structure. The maximum energy of the accelerated particles continues to grow with the duration of the simulation and so does the spatial extent of the precursor, as measured from 
the shock transition. The properties of the leading edge of the precursor also keep evolving in the simulations, e.g., the particle density at the precursor's leading edge keeps dropping as the latter progresses away from the shock transition. This implies that the kinetic instabilities that will develop at the leading edge, on time and length scales that are not sampled by existing simulations, may be very different than those that are observed now in existing simulations.

There are several known instabilities that operate in interpenetrating plasmas (see Bret 2009 for an overview and references). Three specific types of instabilities excited in shock precursors could be important in relativistic unmagnetized or weakly magnetized shocks. These are the kinetic two-stream-like instability, the kinetic filamentation (or transverse Weibel) instability, and the magnetohydrodynamic streaming instability identified by Bell (2004). The Bell instability develops only if the upstream plasma has some pre-existing seed magnetic field. The filamentation instability is suppressed if the accelerated particle precursor is dilute (i.e., its particle density is sufficiently small compared to the upstream plasma density), and its perpendicular momentum spread in the upstream frame is large enough (Silva et al. 2002). The two-stream-like instability, which we define to include the classical two-stream instability as well as its oblique variant, is the only one that grows under any circumstances (at least as long as the upstream plasma remains cold), independent of the density and momentum spread in the upstream frame of the shock precursor. Because of the two-stream-like instability's universal occurrence, we dedicate this work to identifying its fastest growing modes and discussing its potential role in relativistic unmagnetized shocks in astrophysical and numerical settings.

The two-stream-like and filamentation instabilities (sometimes referred to as "Weibel" instability Weibel 1959; Fried 1959; Moiseev \& Sagdeev 1963) arise from the same branch of the dispersion relation and compete with each other (e.g., Bret 2009; Michno \& Schlickeiser 2010). The two-stream parallel unstable mode is found when the wave vector is aligned with the flow, while the filamentation mode has its wave vector perpendicular to the flow. For intermediate orientations, the so-called oblique modes are equally unstable. Since no significant magnetic field is generated, these oblique modes are electrostatic and are very similar to the parallel two-stream mode, explaining why they can be labeled "two-stream-like." For Maxwellian electron beam/plasma system in the non-relativistic regime, two-stream modes grow faster than any other unstable mode. In rather high density relativistic beams, filamentation modes tend to dominate the linear phase. Oblique modes become important in the dilute relativistic beam regime that is the focus of the present work (Faĭnberg et al. 1970; Bret et al. 2008).

Motivated by astrophysical considerations, we consider a relativistic beam that is seen in the rest frame of the background plasma to be having particle Lorentz factors narrowly distributed around $\gamma$, and momenta that are directed within an angle of $\sim \Gamma^{-1}$ from the beam axis, where $\Gamma$ is the shock Lorentz factor. This is an ultrarelativistic beam that is relativistically hot in its own rest frame, namely, $\gamma \gg \Gamma \gg 1$. Two-stream-like filamentation and Buneman modes in this setup (considering both cold and hot upstream plasma) were explored very recently by Lemoine \& Pelletier (2011) (in a previous work, Lemoine \& Pelletier 2010 explored these instabilities in the cold beam cold upstream limit). Lemoine \& Pelletier (2011), however, restrict the investigation of the two-stream-like instability only for modes that satisfy $k_{\|} c=\omega_{\mathrm{pl}} / \beta$, where $\omega_{\mathrm{pl}}$ is the background plasma frequency and $\beta$ is the velocity of beam particles. Here, we explore the entire $\left(k_{\|}, k_{\perp}\right)$ space of the two-streamlike modes (including the pure parallel modes $k_{\perp}=0$ ). We derive the fastest growing mode (which not necessarily satisfies $\left.k_{\|} c=\omega_{\mathrm{pl}} / \beta\right)$ and its growth rate under the assumption that the background plasma remains cold. Our results are applicable to all types of unmagnetized shocks and precursors, including beams containing ions and electrons, ions only, electrons only, or pairs, and upstream plasmas containing ions and electrons or pairs.

We do not go in the present work beyond the linear regime. The nonlinear phase should be explored in the future (possibly with numerical simulations), since it is on the detailed character of the nonlinear development that a possible significant heating by, or momentum transfer from, the accelerated particles is contingent. Similarly, the nonlinear development determines whether the resulting electrostatic field can contribute accelerated particle scattering in the shock upstream.

The paper is organized as follows. In Section 2, we discuss the astrophysical context. In Section 3.1, we define our model distribution function and derive the growth rates of the fastest growing modes. In Section 3.2, we provide a simple physical description of the instability. In Section 4, we discuss possible implications of our results for gamma-ray burst (GRB) external shocks. In Section 5, we provide our conclusions.

\section{THE ASTROPHYSICAL SETTING}

Relativistic collisionless shocks are thought to occur in various astrophysical environments, including in GRBs, pulsar wind nebulae, active galactic nuclei, and microquasars. While the type of plasma (ions and electrons or pairs) and the magnetization level is debated in most of these systems, it is likely that astrophysical relativistic shocks take place in both plasma types and over a large range of magnetization levels. Among these environments, one of the systems where the plasma properties are strongly constrained by observations is the external shock in GRBs. Here an ultrarelativistic blast wave starts with very high Lorentz factors (up to $\Gamma \sim 1000$ and maybe even more) and decelerates with time to Newtonian velocities. It propagates into a very weakly magnetized circum-burst proton-electron plasma (for reviews see Piran 2004; Mészáros 2006; Nakar 2007). Another environment where the plasma type is constrained is the termination shock of pulsar wind nebulae, which is driven into a pair plasma (although some ions may be present), but in this case, the magnetization level of the plasma at the shock is debated (for review see Kirk et al. 2009).

Here, we consider collisionless relativistic shocks of both plasma types, which are non-magnetized or only weakly magnetized. The accelerated particles crossing into the shock upstream constitute a beam of relativistic ions and/or electrons, or relativistic pairs. The acceleration produces a spectrum of energies that is typically approximated with a power law, where the largest number of the accelerated particles, and most of the beam energy contained in the accelerated particles, resides at the low-energy end of the spectrum. Assuming that the particles are accelerated by DSA, the ones that run ahead of the shock must be deflected by interacting with the upstream plasma. Lower energy particles are deflected more easily than those with higher energies. Therefore the particles with higher energies can travel farther ahead of the shock and at any distance from the shock the accelerated beam is dominated, both in particle number and total energy, by the lowest energy particles that reach that distance. 
These are also the particles that we expect to be most susceptible to kinetic instabilities and are thus those that will dominate the growth rate of the two-stream-like instability. ${ }^{5}$ Therefore, we approximate the beam at a given distance from the shock as being monoenergetic with particles Lorentz factor $\gamma$, as observed in the upstream frame, which is the lowest Lorentz factor of beam particles at that distance. The rest frame of the beam, i.e., the frame in which the beam is isotropic, moves with a Lorentz factor $\sim \Gamma$ with respect to the upstream. The beam particles are also highly relativistic in the beam rest frame, implying $\gamma / \Gamma \gg 1$. Thus, we work in the regime in which $\gamma \gg \Gamma \gg 1$.

The charge neutrality of the beam depends on the specific setting. A beam of pairs will always be charge-neutral, but a proton-electron beam may be either charge-neutral or positively charged. If electrons and protons are accelerated with similar efficiencies (as numerical simulations suggest, see, e.g., Spitkovsky 2008a) and radiative losses can be neglected, then at any distance in front of the shock the beam is charge-neutral and contains particles with similar energies (i.e., $m_{e} \gamma_{e} \sim m_{p} \gamma_{p}$ ). Therefore, since in the limit $\gamma_{e}, \gamma_{p} \gg 1$ the beam behavior depends only on the product $m \gamma$, the beam is similar to a beam of pairs. If, on the other hand, electrons are not accelerated as efficiently as protons, either due to less efficient injection into the DSA cycle or due to higher radiative losses, the accelerated particle beam is positively charged. Note that the electrons and protons of an initially cold upstream always behave differently.

An instability can have a strong impact on the structure of an astrophysical system if its amplitude becomes nonlinear. In order to do so it must have enough time to grow, namely, the time that the instability has to grow, $t_{\text {grow }}$, must be significantly longer than the instability growth time, given by the inverse growth rate $1 / \delta$. In the context of our setup, the value of $t_{\text {grow }}$ for an upstream fluid element is the time between the first encounter of the fluid element with the accelerated particle precursor and the time that this element is swept up by the shock transition. If the shock is spherical and its radius is $R$, then $t_{\text {grow }} \lesssim R / c \Gamma^{2}$, where $t_{\text {grow }}$ is measured in the upstream rest frame. In a planar geometry, which is typically used in numerical simulations, $t_{\text {grow }} \leqslant t / \Gamma^{2}$, where $t$ is the age of the shock (both times are measured in the upstream frame). Note that a beam particle spends a time much longer than $t_{\text {grow }}$ in the shock upstream. In spherical geometry it can be as long as $\sim R / c$ and in planar geometry it can be as long as $t$. Therefore, beam particles have much more time to be affected by the interaction with the upstream.

\section{THE FASTEST GROWING MODE}

\subsection{The Dispersion Relation}

Consider a dilute monoenergetic particle beam that propagates in a dense cold plasma. The rest mass of each beam particle is $m_{\mathrm{b}}$ and its energy is $m_{\mathrm{b}} \gamma$, as measured in the background plasma frame. ${ }^{6}$ In this frame the beam momenta are narrowly collimated within a half-opening angle $\sim \Gamma^{-1}$, where

\footnotetext{
5 Our analysis validates this expectation. Below, we find that the instability growth rate is slower for more energetic particles (higher $\gamma$ ) and for beams with lower densities. This result supports our monoenergetic approximation as it implies that particles with much higher Lorentz factor than $\gamma$ are reacting to the background plasma on much longer timescales in the linear regime and can therefore be neglected.

6 If the beam contains several particle species, then we assume energy equipartition between particles of different masses. For example, in a proton-electron beam $m_{e} \gamma_{\mathrm{b}, e}=m_{p} \gamma_{\mathrm{b}, p}\left(\equiv m_{\mathrm{b}} \gamma\right)$. Given the highly relativistic setting considered here, the slight velocity drift between species of different mass (e.g., since $\gamma_{\mathrm{b}, e} \neq \gamma_{\mathrm{b}, p}$ ) can be neglected.
}

$\gamma \gg \Gamma \gg 1$. The following calculation makes no assumptions about the beam and plasma composition.

Since we consider a monoenergetic beam, we can neglect the longitudinal momentum spread of the beam, as seen in the upstream frame, in which the calculations will be conducted. The transverse momentum spread in this frame is $\sim m_{\mathrm{b}} \gamma / \Gamma$. In order to investigate the stability of such a beam, we consider a system containing a beam of density $n_{\mathrm{b}}$ and mean velocity $v_{\mathrm{b}} \approx c$ that interpenetrates a cold background plasma of density $n_{\mathrm{pl}} \gg n_{\mathrm{b}}$. Both densities are measured in the upstream plasma rest frame (in the shock frame the beam density is smaller by a factor of $\Gamma$ while the upstream plasma density is larger by the same factor). The beam density accounts for all species (e.g., in a proton-electron beam, we have $\left.n_{\mathrm{b}}=n_{\mathrm{b}, e}+n_{\mathrm{b}, p}\right)$, while the plasma density includes only the lightest species (e.g., in a proton-electron plasma, we have $n_{\mathrm{pl}}=n_{\mathrm{pl}, e}$ and in a pair plasma, we have $\left.n_{\mathrm{pl}}=n_{\mathrm{pl}, e^{-}}+n_{\mathrm{pl}, e^{+}}\right)$, since the heavier species is assumed to be static. The system is charge-neutral so plasma drift neutralizes the beam charge. In a proton-electron plasma and beam, the electrons drift with velocity $v_{\mathrm{pl}} \approx C\left(n_{\mathrm{b}} / n_{\mathrm{pl}}\right) v_{\mathrm{b}}$ and cancel the beam current, where

$$
C \equiv \frac{n_{\mathrm{b}, p}-n_{\mathrm{b}, e}}{n_{\mathrm{b}}}
$$

is the fractional beam charge satisfying $-1 \leqslant C \leqslant 1$. We show later that this drift has a negligible effect on the instability spectrum and on the growth rate.

Given the symmetry of the dispersion relation with respect to the rotation around the parallel direction, a two-dimensional survey of the $k$-space is required (Bret et al. 2005). In general, such calculation requires solving the linearized Maxwell-Vlasov equations. The electrostatic, or longitudinal, approximation, i.e., $\mathbf{k} \times \mathbf{E}=0$, is not appropriate in general because the modes evolve continuously from $\mathbf{k} \times \mathbf{E}=0$ along the beam direction to $\mathbf{k} \times \mathbf{E} \neq 0$ in the perpendicular direction. For example, the filamentation instability is found for $\mathbf{k} \perp \mathbf{v}_{\mathrm{b}}$ and has $\mathbf{k} \times \mathbf{E} \neq 0$, but it need not have $\mathbf{k} \cdot \mathbf{E}=0$ (Bret et al. 2007). Thus, in general, the electrostatic approximation can lead to a neglect of the most unstable modes. However, in the specific case of interest, where the beam is relativistic and dilute, oblique or parallel parts of the spectrum govern the linear phase of the instability (Bret et al. 2008) and the electrostatic approximation is valid (Bret et al. 2004; Gremillet et al. 2006). Thus, in what follows, we adopt the electrostatic approximation.

The longitudinal dispersion equation thus reads (Ichimaru 1973)

$$
\varepsilon(\mathbf{k}, \omega)=1+\sum_{\sigma} \frac{4 \pi n_{\sigma} e^{2}}{k^{2}} \int \frac{\mathbf{k} \cdot \nabla_{\mathbf{p}} f_{\sigma}(\mathbf{p})}{\omega-\mathbf{k} \cdot \mathbf{v}} d^{3} p=0,
$$

where the sum runs over the species $\sigma$ which are here the beam species, plus the lightest species of the cold background plasma. We now choose the $z$-axis as the direction of beam propagation and label $x$ as the normal direction. The momentum distribution function of each beam species reads

$$
f_{\mathrm{b}}(\mathbf{p})=\frac{1}{2 P_{T}} \delta\left(p_{z}-\gamma m_{\mathrm{b}} v_{\mathrm{b}}\right)\left[\Theta\left(p_{x}+P_{T}\right)-\Theta\left(p_{x}-P_{T}\right)\right],
$$

where $P_{T} \equiv \gamma m_{e} v_{\mathrm{b}} / \Gamma$, while the distribution function of each (background) plasma species is

$$
f_{\mathrm{pl}}(\mathbf{p})=\delta\left(p_{z}+C m_{\mathrm{pl}} v_{\mathrm{pl}}\right) \delta\left(p_{x}\right),
$$


where $m_{\mathrm{pl}}$ is the mass of the lightest plasma particle, typically an electron, and $\Theta(t)$ is the Heaviside step function. Note that $\gamma m_{\mathrm{b}}$ is equal for all beam species and that in a pair plasma $C=0$.

The dispersion equation obtained when inserting these functions in Equation (2) can be written concisely as

$$
0=1-\frac{1}{\left(\tilde{\omega}-C Z_{z} \alpha\right)^{2}}-\alpha \frac{m_{\mathrm{pl}}}{m_{\mathrm{b}}} \Psi
$$

where

$$
\begin{aligned}
\Psi \equiv & \frac{Z_{x}^{2}}{Z_{x}^{2}+Z_{z}^{2}} \frac{\phi(\rho) / \gamma}{\left[Z_{z}-\tilde{\omega} \phi(\rho)\right]^{2}-Z_{x}^{2} \rho^{2}}+\frac{Z_{z}}{2 \gamma^{3}\left(Z_{x}^{2}+Z_{z}^{2}\right)} \\
& \times \int_{-1}^{1} \frac{Z_{z}-\rho t \gamma^{2}\left(Z_{x}-\rho t Z_{z}\right)}{\phi(\rho t)\left[Z_{z}+\rho t Z_{x}-\tilde{\omega} \phi(\rho t)\right]^{2}} d t .
\end{aligned}
$$

Here $\gamma$ is the Lorentz factor of beam particles with mass $m_{\mathrm{b}}$ and we define the following symbols

$\alpha \equiv \frac{n_{\mathrm{b}}}{n_{\mathrm{pl}}}, \mathbf{Z} \equiv \frac{\mathbf{k} c}{\omega_{\mathrm{pl}}}, \rho \equiv \frac{1}{\Gamma}, \tilde{\omega} \equiv \frac{\omega}{\omega_{\mathrm{pl}}}, \phi(\tau) \equiv \frac{1}{\gamma} \sqrt{1+\gamma^{2}\left(1+\tau^{2}\right)}$,

where $\omega_{\mathrm{pl}}^{2}=4 \pi n_{\mathrm{pl}} e^{2} / m_{e}$ is the plasma frequency of the background lightest particles and we approximate $v_{\mathrm{b}} \approx c$. The second term on the right hand side of Equation (5) pertains to the lightest particle species of the background plasma, and the last term accounts for the beam response.

Calculations can be simplified in the present regime noting that $0<\rho \ll 1$ and $\gamma \gg 1$, which implies that $\phi(\rho) \sim 1$ and $\phi(\rho t) \sim 1$ for $-1 \leqslant t \leqslant 1$. The quadrature in Equation (6) can be further simplified to obtain

$$
\Psi \approx \frac{1}{\gamma\left(Z_{x}^{2}+Z_{z}^{2}\right)\left[\left(Z_{z}-\tilde{\omega}\right)^{2}-Z_{x}^{2} \rho^{2}\right]}\left[Z_{x}^{2}+\frac{Z_{z}^{3}}{6 \gamma^{2}} \frac{\left(3+\gamma^{2} \rho^{2}\right)}{\left(Z_{x}^{2}+Z_{z}^{2}\right)}\right] .
$$

All types of beams and plasmas exhibit a similar unstable spectrum. The reason is that the only dimensionless parameter that cannot be removed through scaling is the fractional beam charge $C$. It is the beam charge density that determines whether the background plasma drifts and what is the direction of the drift. However, the drift velocity is proportional to the density ratio $\alpha$ and is thus very small. Therefore in Equation (5), the parameter $C$ is only found in a product with the density ratio $\alpha$ and the effect of space charge in the beam is negligible (note that the value of the third factor in the product is $Z_{z} \sim 1$ for the most unstable mode).

A typical two-dimensional map of the unstable spectrum for a pure electron beam is shown in Figure 1 , for $\alpha=10^{-2}$, $\gamma=100$, and $\Gamma=5$. The growth rate of the fastest growing unstable mode as a function of the mode angle $\tan ^{-1}\left(k_{\perp} / k_{\|}\right)$is shown in Figure 2. The spectrum exhibits two local maxima. One corresponds to the parallel mode with $\left(Z_{\|}, Z_{\perp}\right) \approx(1,0)$ and the other to the oblique mode with $\left(Z_{\|}, Z_{\perp}\right) \approx(1.2,1)$. The relative height of the two maxima, and hence the character of the fastest growing mode-whether it is parallel or oblique, depends on the specific values of the parameters. Also noticeable is the narrowness of the range of wave vectors containing unstable modes. With a high beam Lorentz factor, a mode can only grow out of its interaction with the beam if a certain resonance condition is strictly fulfilled (we discuss this condition

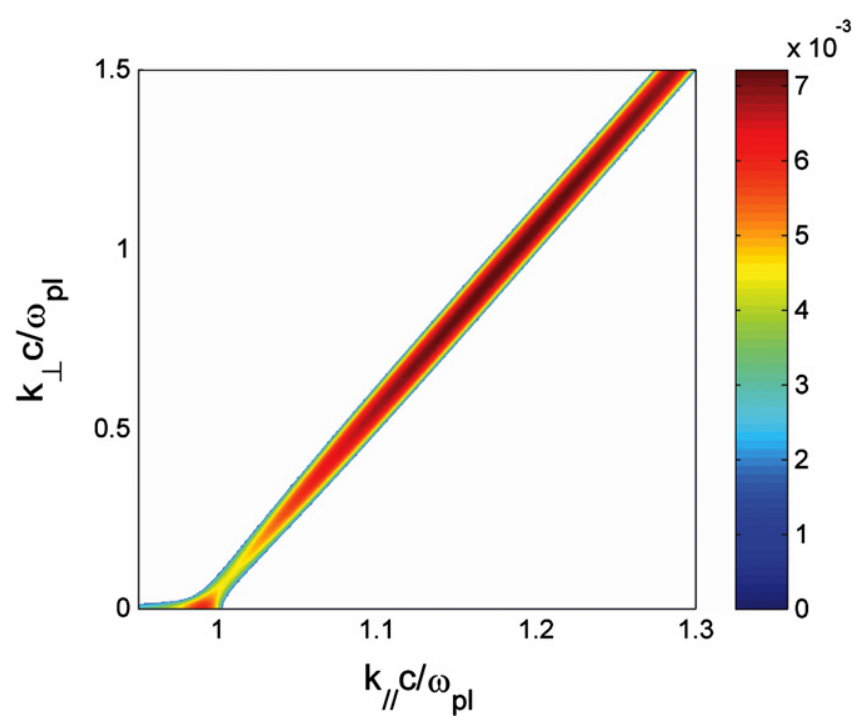

Figure 1. Growth rate in units of the plasma frequency as a function of the dimensionless wave vector $\mathbf{Z}=\mathbf{k} c / \omega_{\mathrm{pl}}$, in the case of a pure electron beam. Parameters are $\alpha=10^{-2}, \gamma=100$, and $\Gamma=5$.

(A color version of this figure is available in the online journal.)

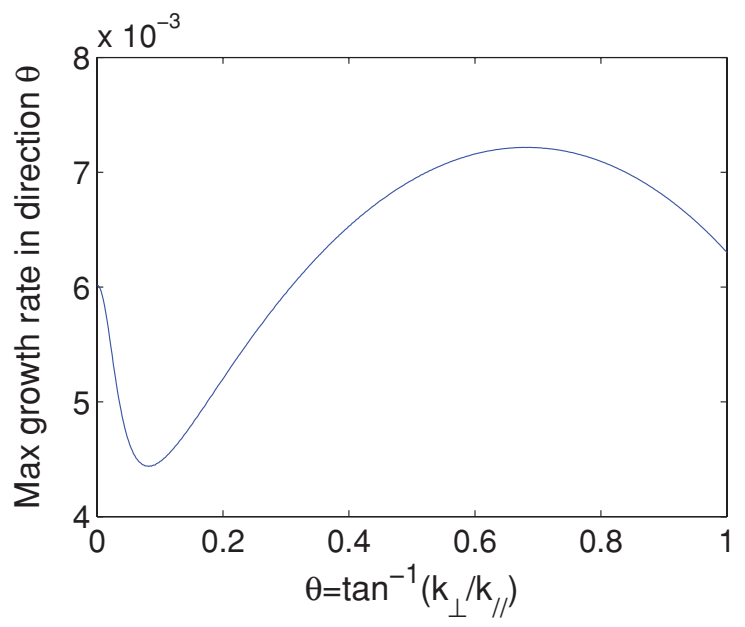

Figure 2. Growth rate in units of the plasma frequency as a function of the mode angle $\tan ^{-1}\left(k_{\perp} / k_{\|}\right)$. Parameters are the same as in Figure 1.

(A color version of this figure is available in the online journal.)

in Section 3.2). Modes which are "out of tune" are quickly damped away.

The total absence of unstable modes in the perpendicular direction is not always just a direct consequence of the electrostatic approximation, which does not account for such nonlongitudinal modes. The theory of unstable filamentation modes for the present setting shows that they are completely shut down if the transverse beam angular spread as seen in the upstream frame $\sim \Gamma^{-1}$ is larger than $\left[\alpha m_{\mathrm{pl}} /\left(\gamma m_{\mathrm{b}}\right)\right]^{1 / 2}$, that is, when the density ratio is limited by (Silva et al. 2002; Bret et al. 2005; Rabinak et al. 2010)

$$
\alpha<\frac{\gamma m_{\mathrm{b}}}{\Gamma^{2} m_{\mathrm{pl}}} \text { (filamentation suppression). }
$$

Since we work here in the regime in which $\alpha \ll 1 \ll \Gamma \ll \gamma$, filamentation modes are unstable only in a small portion of the phase space, especially in an ion-electron plasma. Moreover, if the first shock-crossing cycle of the DSA accelerates particles to Lorentz factors $\gamma \sim \Gamma^{2}$ (e.g., Gallant \& Achterberg 1999), then the filamentation modes are stable in the entire precursor 
for any type of plasma. Finally, let us emphasize that even when filamentation modes are unstable, their growth rate is slower than the fastest growing two-stream mode that we find here, so they do not dominate anyway.

The growth rate of the fastest purely parallel growing mode can be found analytically. The dispersion equation for such wave vectors, $\mathbf{Z}=Z_{z} \hat{\mathbf{z}}$, is easily derived from Equations (2) and (6) and reads

$$
0=1-\frac{1}{\left(\tilde{\omega}-C Z_{z} \alpha\right)^{2}}-\alpha \frac{m_{\mathrm{pl}}}{m_{\mathrm{b}}} \frac{\left(3+\gamma^{2} \rho^{2}\right)}{3\left(\tilde{\omega}-Z_{z}\right)^{2} \gamma^{3}} .
$$

This relation is mathematically similar to the dispersion relation of the two-stream instability. The maximum parallel growth rate $\delta_{\text {par }}$ is reached for $Z_{z} \sim 1$ and reads

$$
\delta_{\mathrm{par}} \approx \frac{3^{1 / 6}}{2^{4 / 3}}\left(\frac{m_{\mathrm{pl}}}{m_{\mathrm{b}}} \frac{\alpha}{\gamma \Gamma^{2}}\right)^{1 / 3} \omega_{\mathrm{pl}} .
$$

The growth rate of the fastest growing oblique mode can be solved for numerically. We conducted a systematic numerical search for the fastest growing mode for representative choices of $\alpha$, $\gamma$, and $\Gamma$. We find that when $\alpha \ll 1$ and $1 \ll \Gamma \ll \gamma$, the maximum oblique growth rate $\delta_{\text {obl }}$ reads

$$
\delta_{\mathrm{obl}} \approx \frac{1}{3}\left(\frac{m_{\mathrm{pl}}}{m_{\mathrm{b}}} \frac{\alpha \Gamma}{\gamma}\right)^{1 / 2} \omega_{\mathrm{pl}}
$$

Equating the two growth rates yields the criterion for the dominance of each, so that the global fastest growing mode grows at the rate

$$
\delta_{\max }= \begin{cases}\delta_{\mathrm{par}} ; \frac{2^{8 / 7}}{3}\left(\frac{m_{\mathrm{pl}} \alpha}{m_{\mathrm{b} \gamma} \gamma}\right)^{1 / 7} & \Gamma<1, \\ \delta_{\mathrm{obl}} ; \frac{2^{8 / 7}}{3}\left(\frac{m_{\mathrm{pl}} \alpha}{m_{\mathrm{b} \gamma} \gamma}\right)^{1 / 7} & \Gamma>1 .\end{cases}
$$

Some of the general trends evident in Equations (11) and (12) were to be expected. Namely, the growth rate weakens when the beam density decreases or when the inertia of the beam particles increases. The fact that the growth rate has two local maxima with opposite dependencies on $\Gamma$ is nontrivial and is discussed in the next section. It is interesting to compare this result to the scenario in which a cold beam interacts with a cold plasma (Faĭnberg et al. 1970). There, the fastest growing mode is always oblique and its growth rate scales as $(\alpha / \gamma)^{1 / 3}$.

Finally, we stress that Equations (11) and (12) provide the growth rates for monoenergetic beam with an idealized distribution function given in Equation (3). The true distribution function in collisionless shocks with DSA is of course more complicated. Since the exact value of the growth rate and its dependence on the various parameters depend on the exact shape of the distribution function, we expect the result to be somewhat different if a more realistic distribution is considered. However, we still expect that the key proportionalities evident in Equations (11) and (12) persist in the general case, although the exact values of the power-law indices may vary. We also expect that there will still be a competition between the parallel and oblique modes.

\subsection{Intuitive Physical Description}

The oblique mode is a two-stream-like electrostatic mode, and as such it can be understood with the aid of the intuition gained

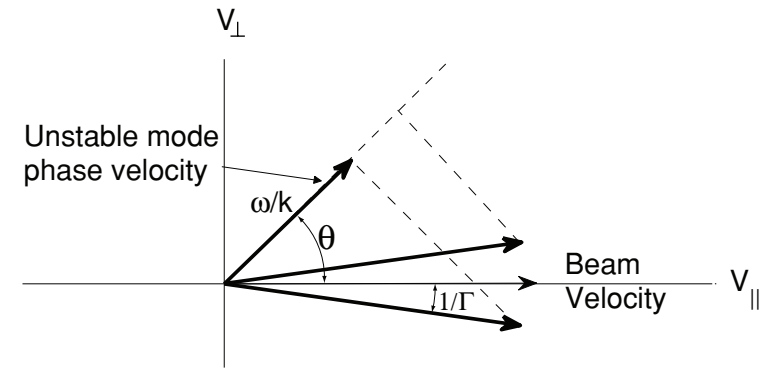

Figure 3. Illustration of the beam and wave mode geometry.

from the consideration of a one-dimensional, longitudinal twostream mode. In one dimension, a wave mode interacts with near-resonant particles that move with velocities similar to the phase velocity of the mode. These particles "surf" the wave in the sense that they spend a long time in phase with the wave. During surfing, the velocity of near-resonant particles further converges to the phase velocity of the wave. The particles that are slightly faster than the wave tend to transfer energy to the wave, and thus excite it, while those that are slightly slower than the wave tend to receive energy from it, and thus damp the wave. Since growing modes are those with a net energy gain, a mode is unstable if the number of particles moving slightly faster than the wave exceeds that of those moving slightly slower. Thus, a distribution function $f(v)$ should be two-stream unstable only if $(\partial f / \partial|v|)_{v_{\text {phase }}}>0$, where $v_{\text {phase }}=\operatorname{Re}(\omega) / k$ is the phase velocity of the wave mode.

The three-dimensional case is similar, except that now it is $v_{\mathbf{k}}$, the component of the velocity parallel to the wave vector $\mathbf{k}$ that determines the stability of the mode. In the configuration under consideration all the accelerated particles move at about the speed of light and have momenta that are collimated to within the small angle $\sim \Gamma^{-1}$ from the direction of shock propagation (see Figure 3). For any generic wave vector, except for the almost perpendicular ones, the range of particle velocities projected onto the wave vector does not include the zero velocity. Thus, a mode with phase velocity equal to the minimum of the projected beam particle velocities has many particles that are moving slightly faster than the wave but none that are moving slower than the wave. Based on the one-dimensional analysis in the preceding paragraph, the mode is unstable. Neighboring modes with an excess of particles moving faster than the wave should also be unstable. All unstable two-stream oblique modes satisfy the resonance criterion

$$
\frac{v_{\text {phase }}}{c} \equiv \frac{\operatorname{Re}(\omega)}{c k} \approx \cos \left(\theta+\Gamma^{-1}\right),
$$

where $\theta$ is the angle between $\mathbf{k}$ and the $z$-axis. This includes the parallel mode, $\theta=0$, and oblique modes up to $\theta=$ $\pi / 2-\Gamma^{-1}$. Figure 4 shows a growth rate map in the plane of $\frac{\operatorname{Re}(\omega)}{c k}\left[\cos \left(\theta+\Gamma^{-1}\right)\right]^{-1}$ and $\theta$, where the resonance is clearly evident. The resonance condition for instability in the regime that we consider, up to terms of the order of $\Gamma^{-2}$, can therefore be written as

$$
\operatorname{Re}(\omega) \approx c k_{\|}-\frac{c k_{\perp}}{\Gamma} .
$$

This intuitive description does not select the fastest growing mode among all the unstable modes. In Section 3.1, we analyzed the dispersion relation to pin down the dominant mode and derived the dependence of growth rate on the shock Lorentz factor $\Gamma$ in the parallel and oblique regimes. The different 


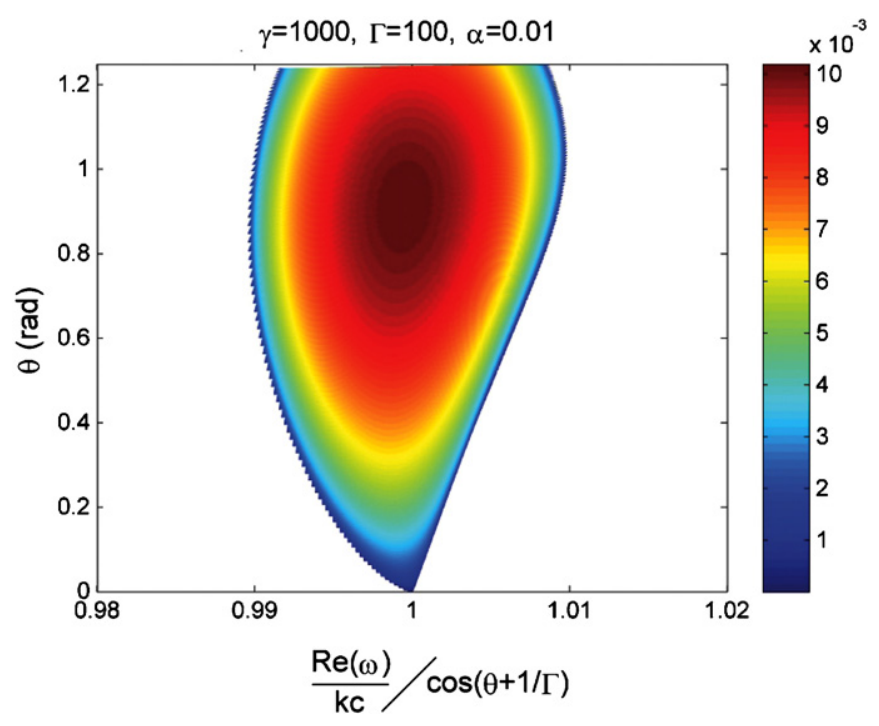

Figure 4. Growth rate map in the plane defined by $\frac{\operatorname{Re}(\omega)}{c k}\left[\cos \left(\theta+\Gamma^{-1}\right)\right]^{-1}$ and $\theta$ for unstable modes with $0<Z_{\|}<2$ and $0<Z_{\perp}<3$. The color shows the growth rate of a pure electron beam with the parameters $\alpha=10^{-2}, \gamma=1000$, and $\Gamma=100$. The narrow concentration of the unstable modes around the resonant condition $\frac{\operatorname{Re}(\omega)}{c k}\left[\cos \left(\theta+\Gamma^{-1}\right)\right]^{-1}=1$ is clearly evident.

(A color version of this figure is available in the online journal.)

dependencies of the growth rates on $\Gamma$ can be understood as follows. In general, the growth rate depends on two competing and opposite factors. One is the number of particles that are in resonance with the mode. The energy of these particles can in principle be tapped toward contributing to the growth of the mode. The other relates to the efficiency with which energy transfer from the particles to the mode can take place. The second factor is the reason that oblique modes play an important role in relativistic beams. It is much easier to reduce the projected velocity of an ultrarelativistic particle in the direction of $\mathbf{k}$, and thus to trap the particle in a resonance with the mode and transfer its energy to the mode, by deflecting the particle sideways, than by decelerating the particle. From this it follows that a larger value of the angle between the resonant particles and the wave, $\theta+\Gamma^{-1}$, contributes to increasing the growth rates. On the other hand, now returning to the first aforementioned factor, larger values of $\Gamma$ and smaller values of $\theta$ imply a distribution function that is more compact when projected onto the direction of $\mathbf{k}$. This ensures that a larger number of nearly resonant particles is available. From this, however, it follows that having a larger value of $\theta+\Gamma^{-1}$ also works in the opposite direction, namely, toward decreasing the growth rates. It is the balance between these to opposite dependencies of the two effects on $\theta+\Gamma^{-1}$ that determines the location in $\mathbf{k}$ space of the fastest growing mode. Now, in oblique modes, where $\theta \gg \Gamma^{-1}$ and thus $\theta+\Gamma^{-1} \approx \theta$, increasing $\Gamma$ only increases the compactness of the distribution function in the direction of $\mathbf{k}$, thereby increasing the growth rate of the fastest growing oblique mode. In parallel modes where $\theta \approx 0$ and thus $\theta+\Gamma^{-1} \approx \Gamma^{-1}$, increasing $\Gamma$ increases the number of resonant beam particles but makes it harder to exploit their energy. It turns out that the latter factor wins and the growth rate increases when $\Gamma$ is smaller.

\section{APPLICATION TO EXTERNAL SHOCKS IN GRBs}

Here, we assess under which conditions the two-stream-like instability in the precursor of GRB external shocks can become nonlinear and strongly affect the shock structure, assuming that the acceleration process is DSA. We do so by comparing $t_{\text {grow }}$ in such a shock (see the definition in Section 2 ) to $1 / \delta_{\max }$ found in Section 3.1. A GRB external shock is a spherical relativistic collisionless shock that propagates in a weakly magnetized proton-electron plasma. Electron acceleration in GRB external shocks is expected to be limited by the radiative cooling ( $\mathrm{Li}$ $\&$ Waxman 2006; Couch et al. 2008) and thus the precursor beam should contain mostly protons at higher energies. The accelerated particle spectrum is taken to be a power law $d N_{\text {acc }} / d \gamma \propto \gamma^{-p}$ where $N_{\text {acc }}$ is the total number of accelerated particles in the shocked region and the power-law index is in the range $2<p<3$. We assume that the minimum accelerated particle energy is $\sim \Gamma^{2}$, where $\Gamma$ is the shock Lorentz factor, as expected for the accelerated particles that have completed their first full Fermi cycle. The density of the accelerated particle precursor can then be parameterized by the fraction $\epsilon_{\text {acc }}$ of the shock energy that goes into the accelerated particles. The total accelerated particle number is then $N_{\text {acc }} \sim \epsilon_{\text {acc }} E /\left(\Gamma^{2} m_{p} c^{2}\right)$, where $E$ is the total shock energy. The density of accelerated particles with Lorentz factors $\sim \gamma$, as measured in the upstream frame, is $n_{\mathrm{acc}}(\gamma) \sim\left(\Gamma^{2} / R^{3}\right)\left(\gamma / \Gamma^{2}\right)^{1-p} N_{\mathrm{acc}}$, where $R$ is the shock radius. We assume that the density $n$ of the unshocked upstream is constant. The hydrodynamics of an ultrarelativistic blast wave (e.g., Blandford \& McKee 1976) relate $n$ to the blast wave energy $E$, radius $R$, and Lorentz factor $\Gamma$; with this, one finds that the density ratio measured in the upstream frame, $\alpha \equiv n_{\text {acc }} / n$, is

$$
\alpha(\gamma) \sim 0.1\left(\frac{\epsilon_{\mathrm{acc}}}{0.1}\right)\left(\frac{\Gamma}{100}\right)^{2 p}\left(\frac{\gamma}{10^{6}}\right)^{1-p} .
$$

Note that $\alpha$ retains no explicit dependence on $E$ or $n$. At any distance from the shock the accelerated particle density is dominated by the lowest energy particles that reach that distance.

We first determine which among the oblique and parallel modes dominates. Substituting Equations (16) into (13), using $m_{\mathrm{b}}=m_{p}$, we find that with $\gamma \sim 10^{6}$ (the dependence on the particle Lorentz factor is weak), for $\Gamma \gtrsim 60$ the oblique mode dominates while for $\Gamma \ll 60$ the parallel mode dominates. Since most of the afterglow observations cover the evolution when $\Gamma<60$, in what follows, we will focus on the low- $\Gamma$ regime in which the parallel mode dominates. The growth rate derived in Equation (11) decreases with increasing $\gamma$ and scales as $\delta_{\max } \propto\left(\alpha / \gamma \Gamma^{2}\right)^{1 / 3} \propto \gamma^{-p / 3} \Gamma^{2(p-1) / 3}$. The time available for growth is $t_{\text {grow }} \sim \Delta(\gamma) / c$ where $\Delta(\gamma)$ is the distance from the shock that particles with Lorentz factors $\gamma$ can reach. The maximum distance is $\Delta_{\max } \sim R /\left(10 \Gamma^{2}\right)$, and it is reached by the protons that are accelerated to the highest energies $\gamma_{\max }$. A pre-existing shock upstream magnetic field with a strength of a few $\mu \mathrm{G}$ and with a coherence length that is larger than the shock radius, as expected in the interstellar medium (ISM), can accelerate protons up to $\gamma_{\max } \sim 10^{6}$. If accelerated particle streaming in the shock precursor can somehow amplify the preexisting field to the point of equipartition with the rest energy of the circum-burst medium, proton acceleration to energies $\gamma_{\max } \sim 10^{10}$ may be possible (Milosavljević \& Nakar 2006). We approximate the dependence of the distance from the shock on the particle Lorentz factor with a power law $\Delta(\gamma) \propto \gamma^{s}$. If all the accelerated particles are deflected back into the shock by a magnetic field with a coherence length larger than $R$, then we have $s=1$. If, on the other hand, they are deflected by a small scale with coherence length $\lambda_{B} \ll R$, then we have $s=2$ at all distances $\Delta>\lambda_{B}$. 
The maximum number of $e$-foldings by which an unstable mode can grow is the ratio $t_{\text {grow }} / \delta_{\max }^{-1}$, implying that $N_{e-\text { fold }}(\gamma)=\delta_{\max }(\gamma) \Delta(\gamma) / c$. Using Equations (11) and (16), we find

$$
\begin{aligned}
N_{e \text {-fold }}(\gamma) \approx & 100\left(\frac{\epsilon_{\mathrm{acc}}}{0.1}\right)^{1 / 3}\left(\frac{\Gamma}{100}\right)^{(2 p-10) / 3}\left(\frac{n}{1 \mathrm{~cm}^{-3}}\right)^{1 / 6} \\
& \times\left(\frac{E}{10^{53} \mathrm{erg}}\right)^{1 / 3}\left(\frac{\gamma_{\max }}{10^{6}}\right)^{-p / 3}\left(\frac{\gamma}{\gamma_{\max }}\right)^{s-p / 3} .
\end{aligned}
$$

The dependence on $E$ and $n$ is rather weak, while the scaling with $\Gamma^{-1}$ varies from being linear to being quadratic for $2<p<3$. This implies that if the accelerated protons carry a non-negligible fraction of the total energy of the shock, e.g., $\epsilon_{\text {acc }} \gtrsim 10^{-3}$, but the maximum proton energy is relatively low, $\gamma_{\max } \lesssim 10^{6}$, then as long as the external shock remains relativistic $N_{e-\text { fold }}\left(\gamma_{\max }\right) \gg 1$, and at the largest distance from the shock there is enough time for the two-stream modes to become nonlinear, then the instability can strongly influence the state of the upstream plasma and accelerated particle beam dynamics. If, in the other extreme, $\gamma_{\max } \sim 10^{10}$ and $s=1$, then $N_{e-\text { fold }}\left(\gamma_{\max }\right) \lesssim 1$ throughout the external shock, and the twostream instability should not affect its structure. For intermediate values of $\gamma_{\max }$ or when $s=2$, the number of $e$-foldings is below unity when $\Gamma$ is large, but it can become larger than unity as $\Gamma$ decreases; this transition may imply a change in the shock structure. Finally, we note that for low values of $\gamma$, i.e., close to the shock, and for high values $\Gamma$, it is possible that the accelerated particle density exceeds the upstream plasma density, $\alpha>1$, in which case our analysis is no longer valid.

\section{SUMMARY}

We explored the linear growth rate of electrostatic twostream-like modes that grow when a dilute ultrarelativistic beam, which is relativistically hot in its own rest frame, propagates in a cold, unmagnetized or weakly magnetized, background plasma. This beam-plasma configuration describes the precursor containing accelerated particles that runs ahead of an ultrarelativistic, unmagnetized or weakly magnetized, collisionless shock. We considered various types of plasma (electrons and ions or pairs) and beams of various compositions and studied the fastest growing kinetic modes. Such modes may thus be the principal agent of nonlinear precursor-upstream interaction that is expected in these shocks and that is also apparent in numerical simulations (e.g., Keshet et al. 2009).

Our main finding is that for any beam and plasma composition and for any shock and beam parameters, as long as $\gamma \gg \Gamma \gg 1$ and the background plasma is cold, there exist two-stream-like unstable modes. These are the modes that satisfy the resonance criterion $\operatorname{Re}(\omega) / c k \approx \cos \left(\theta+\Gamma^{-1}\right)$, where $\theta$ is the angle between wave vector $\mathbf{k}$ and the direction of beam propagation. This includes the parallel mode with $\theta=0$, and oblique modes up to $\theta=\pi / 2-\Gamma^{-1}$. The resonance criterion can also be written as $\operatorname{Re}(\omega) \approx c k_{\|}-c k_{\perp} / \Gamma$. This behavior departs from that of the filamentation (or transverse Weibel) modes, which are suppressed over a large range of the parameter space. Moreover, in the interaction of a dilute accelerated particle precursor with a dense, cold, unmagnetized, and unperturbed upstream, the fastest growing electrostatic two-stream-like mode always grows faster than any of the filamentation modes when the latter are unstable. We therefore do not expect filamentation modes to play a role in the shock precursor containing accelerated particles, but possibly only in the shock transition itself.
We find that the spectrum of unstable electrostatic modes exhibits two local maxima. One contains parallel modes, with wave vectors $\left(k_{\|}, k_{\perp}\right) \sim \frac{\omega_{\mathrm{pl}}}{c}(1,0)$, and the other contains oblique ones with $\left(k_{\|}, k_{\perp}\right) \sim \frac{\omega_{\mathrm{pl}}}{c}\left(1+\Gamma^{-1}, 1\right)$. The relative height of the two maxima, and therefore the character of the dominant mode-whether it is of the parallel or oblique type-depends on the parameters and is most sensitive to $\Gamma$. For large values of $\Gamma$, an oblique mode dominates, while for small values of $\Gamma$, it is the parallel mode that dominates. The criterion for finding the dominant mode exhibits a similar trend with $m_{\mathrm{pl}} \alpha /\left(m_{\mathrm{b}} \gamma\right)$, but this dependence is much weaker. Nevertheless, in pair or electron beams, the oblique mode dominates over a larger portion of the parameter space than it does in ion beams.

The time that is available to an unstable mode for its growth is limited in the precursor of astrophysical ultrarelativistic shocks. Nevertheless, if sufficient time is available for the fastest growing mode to become nonlinear, the nonlinear outcome of the instability may significantly affect the shock structure. It can lead to momentum transfer from the accelerated particles to the shock upstream, thereby modifying the hydrodynamic structure of the shock. We compared the growth time of the fastest growing two-stream-like mode to the time available for its growth in GRB external shocks. We find that for all except for the earliest phases, when the shock Lorentz factor has dropped below $\sim 100$, the parallel mode dominates. If the maximum Lorentz factor of the accelerated protons is modest, $\gamma_{\max } \sim 10^{6}$, then this mode has enough time to become nonlinear, while if $\gamma_{\max }$ is significantly larger, then the mode may become nonlinear only for relatively low values of $\Gamma$. We conclude that the electrostatic two-stream-like instability may play an important role in accelerating particles and in shaping of the structure of the external shocks in GRBs.

We thank Uri Keshet and Anatoly Spitkovsky for helpful discussions. E.N. was supported in part by the Israel Science Foundation (grant no. 174/08) and by an EU International Reintegration Grant. A.B. was supported by projects ENE200909276 of the Spanish Ministerio de Educación y Ciencia and PEII11-0056-1890 of the Consejería de Educación y Ciencia de la Junta de Comunidades de Castilla-La Mancha.

\section{REFERENCES}

Achterberg, A., Gallant, Y. A., Kirk, J. G., \& Guthmann, A. W. 2001, MNRAS, 328,393

Bell, A. R. 1978, MNRAS, 182, 147

Bell, A. R. 2004, MNRAS, 353, 550

Blandford, R., \& Eichler, D. 1987, Phys. Rep., 154, 1

Blandford, R. D. 1980, ApJ, 238, 410

Blandford, R. D., \& McKee, C. F. 1976, Phys. Fluids, 19, 1130

Blandford, R. D., \& Ostriker, J. 1978, ApJ, 221, L29

Bret, A. 2009, ApJ, 699, 990

Bret, A., Firpo, M.-C., \& Deutsch, C. 2004, Phys. Rev. E, 70, 046401

Bret, A., Firpo, M.-C., \& Deutsch, C. 2005a, Phys. Rev. Lett., 94, 115002

Bret, A., Firpo, M.-C., \& Deutsch, C. 2005b, Phys. Rev. E, 72, 016403

Bret, A., Gremillet, L., \& Bellido, J. C. 2007, Phys. Plasmas, 14, 032103

Bret, A., Gremillet, L., Bénisti, D., \& Lefebvre, E. 2008, Phys. Rev. Lett., 100, 205008

Couch, S. M., Milosavljević, M., \& Nakar, E. 2008, ApJ, 688, 462

Drury, L. O., \& Voelk, J. H. 1981, ApJ, 248, 344

Ellison, D. C., \& Double, G. P. 2002, Astropart. Phys., 18, 213

Faĭnberg, Y. B., Shapiro, V. D., \& Shevchenko, V. 1970, Sov. Phys., JETP, 30, 528

Fried, B. D. 1959, Phys. Fluids, 2, 337

Gallant, Y. A., \& Achterberg, A. 1999, MNRAS, 305, L6

Gremillet, L., Bénisti, D., Bret, A., \& Lefebvre, E. 2007, Phys. Plasmas, 14, 040704 
Ichimaru, S. 1973, Basic Principles of Plasma Physics (Reading, MA: Benjamin) Keshet, U., Katz, B., Spitkovsky, A., \& Waxman, E. 2009, ApJ, 693, L127

Kirk, J. G., Lyubarsky, Y., \& Petri, J. 2009, in Neutron Stars and Pulsars, ed. W. Becker (Astrophysics and Space Science Library, Vol. 357; Berlin: Springer), 421

Lemoine, M., \& Pelletier, G. 2010, MNRAS, 402, 321

Lemoine, M., \& Pelletier, G. 2011, arXiv:1102.1308

Li, Z., \& Waxman, E. 2006, ApJ, 651, 328

Martins, S. F., Fonseca, R. A., Silva, L. O., \& Mori, W. B. 2009, ApJ, 695, L189

Mészáros, P. 2006, Rep. Prog. Phys., 69, 2259

Michno, M. J., \& Schlickeiser, R. 2010, ApJ, 714, 868
Milosavljević, M., \& Nakar, E. 2006, ApJ, 651, 979

Moiseev, S. S., \& Sagdeev, R. Z. 1963, J. Nucl. Energy C, 5, 43

Nakar, E. 2007, Phys. Rep., 442, 166

Niemiec, J., \& Ostrowski, M. 2006, ApJ, 641, 984

Piran, T. 2004, Rev. Mod. Phys., 76, 1143

Rabinak, I., Katz, B., \& Waxman, E. 2010, arXiv:1005.3791

Silva, L. O., Fonseca, R. A., Tonge, J. W., Mori, W. B., \& Dawson, J. M. 2002, Phys. Plasmas, 9, 2458

Spitkovsky, A. 2008a, ApJ, 673, L39

Spitkovsky, A. 2008b, ApJ, 682, L5

Webb, G. M., Axford, W. I., \& Terasawa, T. 1983, ApJ, 270, 537

Weibel, E. S. 1959, Phys. Rev. Lett., 2, 83 